\title{
Glycaemic index and perceived satiety following ingestion of sourdough breads enriched with soluble fibres
}

\author{
B. Buczkowski \\ Manchester Metropolitan University, Department of Food and Tourism Management, Hollings Faculty, \\ Cavendish Building, Cavendish Street, Manchester, M15 6BG
}

High-glycaemic index (high-GI) diets have been linked to the prevalence of type II diabetes ${ }^{(1-3)}$, coronary heart mortality, certain types of cancer and elevated blood levels of triglycerides and LDL cholesterol ${ }^{(3)}$. Previously, sourdough was successfully used in development of low GI wheat bread ${ }^{(4)}$ and was demonstrated to produce improved glycaemic response even in subjects with impaired glucose tolerance ${ }^{(5)}$. Additionally, viscous soluble fibre may reduce postprandial glycaemia ${ }^{(6)}$. Despite the fact that soluble fibres were shown to produce prolonged satiety ${ }^{(7,8)}$, the findings of studies concerning the satietogenic properties of carbohydrate remain inconclusive $^{(9)}$. The aim of this study was to assess the glycaemic and satietogenic properties of sourdough bread enriched with soluble fibres, control sourdough bread, and white wheat bread.

The glycaemic and satietogenic properties of sourdough bread enriched with soluble fibres (XG/GA/Pec), control sourdough bread and white wheat bread (WWB) were tested in a cross-over study using 11 healthy participants (mean age $35 \pm 10$ years, BMI $23.7 \pm$ $2.86 \mathrm{~kg} / \mathrm{m}^{2}$ ), a standard seven-point protocol and Satiety Labelled Intensity Magnitude (SLIM) scale.

\begin{tabular}{|c|c|c|c|c|}
\hline & \multicolumn{2}{|c|}{ Glycaemic index } & \multicolumn{2}{|c|}{ iAUC of satiety response } \\
\hline & Mean & $\mathrm{SE}$ & Mean & SE \\
\hline Control sourdough & $65 \cdot 70^{\mathrm{d}}$ & $9 \cdot 56$ & $6407 \cdot 96^{\mathrm{c}, \mathrm{d}}$ & 591.41 \\
\hline $\mathrm{XG} / \mathrm{GA} / \mathrm{Pec}$ & $58.93^{\mathrm{d}}$ & $8 \cdot 85$ & $6799 \cdot 77^{\mathrm{c}, \mathrm{d}}$ & $752 \cdot 24$ \\
\hline WWB & $69.83^{\mathrm{d}}$ & $7 \cdot 79$ & $4459 \cdot 81^{\mathrm{a}, \mathrm{b}}$ & $487 \cdot 34$ \\
\hline Glucose & $100^{\mathrm{a}, \mathrm{b}, \mathrm{c}}$ & $2 \cdot 34 \mathrm{E}-15$ & $2401 \cdot 31^{\mathrm{a}, \mathrm{b}}$ & $655 \cdot 92$ \\
\hline
\end{tabular}

The differences of values with different superscripts in columns are statistically significant $(p<0 \cdot 05)$.

GI values were 66 for control sourdough bread $(p=0 \cdot 03), 59$ for XG/GA/Pec $(p=0.006)$ and 70 for white sliced bread $(p=0 \cdot 019)$ with glucose as reference food $(\mathrm{GI}=100)$. After 120 minutes from ingestion, subjects reported higher satiety after control sourdough bread $(p=0.027)$ and sourdough with XG/GA/Pec $(p=0.001)$ than after glucose. Additionally, the bread with XG/GA/Pec was perceived by the subjects to be more satiating after 120 minutes than WWB $(p=0.036)$. iAUC for control sourdough bread and XG/GA/ Pec was higher than those of glucose $(p=0.018$ and $p=0.007$ respectively) and WWB $(p=0.045$ and $p=0.036$ respectively). The value of iAUC of XG/GA/Pec was higher than that of control sourdough bread. This result did not reach statistical significance in statistical analysis. In conclusion, sourdough bread and sourdough bread enriched with soluble fibres were characterised by increased perceived satiety. Delayed gastric emptying is a plausible explanation of the satietogenic properties of sourdough breads enriched with soluble dietary fibre.

This study was conducted according to The Declaration of Helsinki.

Acknowledgements: Dr Joanna Kershaw, Dr Emma Derbyshire, Dr Weili Li and Prof. Charles Brennan. The author would like to acknowledge the help of Prof. Glyn Phillips.

1. Salmerón J, Ascherio A, Rimm EB et al. (1997) Diabetes Care 20, 545-550.

2. Salmerón J, Manson JE, Stampfer MJ et al. (1997) J Am Med Assoc 277, 472-477.

3. Brand-Miller JC (2003) Nutr Rev 61, S49-S55.

4. De Angelis M, Damiano N, Rizello CG et al. (2009) Eur Food Res Technol 229, 593-601.

5. Maioli M, Pes GM, Sanna M et al. (2008) Acta Diabetol 45, 91-96.

6. Brennan MA, Derbyshire E, Tiwari BK et al. (2012) J Agric Food Chem 60, 4396-4401.

7. Di Lorenzo C, Williams CM, Hajnal F et al. (1988) Gastroenterology 95, 1211-1215.

8. Calame W, Thomassen F, Hull S et al. (2011) Appetite 57, 358-364.

9. Anderson GH \& Woodend D (2003) Nutr Rev 61, S17-S26. 